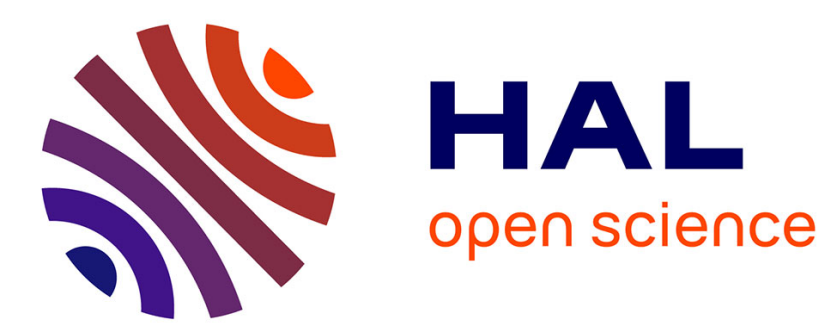

\title{
Continuum Approximation for Congestion dynamics along freeway Corridors
}

\author{
J. Laval, L. Leclercq
}

\section{To cite this version:}

J. Laval, L. Leclercq. Continuum Approximation for Congestion dynamics along freeway Corridors. Transportation Science, 2010, Vol44, n1, p87-97. hal-00506614

\section{HAL Id: hal-00506614 https://hal.science/hal-00506614}

Submitted on 28 Jul 2010

HAL is a multi-disciplinary open access archive for the deposit and dissemination of scientific research documents, whether they are published or not. The documents may come from teaching and research institutions in France or abroad, or from public or private research centers.
L'archive ouverte pluridisciplinaire HAL, est destinée au dépôt et à la diffusion de documents scientifiques de niveau recherche, publiés ou non, émanant des établissements d'enseignement et de recherche français ou étrangers, des laboratoires publics ou privés. 


\title{
2 Continuum Approximation for Congestion Dynamics Along Freeway Corridors
}

\author{
Jorge A. Laval \\ School of Civil and Environmental Engineering, Georgia Institute of Technology, jorge.laval@ce.gatech.edu, \\ http://trafficlab.ce.gatech.edu/ \\ Ludovic Leclercq \\ Université de Lyon - ENTPE / INRETS - Laboratoire Ingénierie Circulation Transport,leclercq@entpe.fr,
}

\begin{abstract}
In this paper, congestion dynamics along crowded freeway corridors are modeled as a conservation law with a source term that is continuous in space. The source term represents the net inflow from ramps, postulated here as a location-dependent function of the demand for entering and exiting the corridor. Demands are assumed time-independent, which is appropriate for understanding the onset of congestion. Numerical and analytical results reveal the existence of four well-defined regions in time-space, two of which are transient. The conditions for the existence of congestion both in the freeway and in the on-ramps are identified, as well as the set of on-ramps that are most likely to become active bottlenecks. The results in this paper help explain the stochastic nature of bottleneck activation, and can be applied to devise effective system-wide ramp metering strategies that would prevent excessively long on-ramp queues.
\end{abstract}

Key words: Continuum Approximation; kinematic wave model; traffic dynamics; congestion

\section{Introduction.}

The main drawback of current traffic flow network models based on the kinematic wave model (Lighthill and Whitham 1955, Richards 1956) is the treatment of boundary conditions at merges. Invariably, it is assumed full priority for the entering flows, which is convenient for mathematical tractability but only reasonable under very light traffic (see e.g. Coclite and Piccoli 2002, Bayen et al. 2004, Gugat et al. 2005, Coclite et al. 2005, Bastin et al. 2007, Jin and Zhang 2003, 2004). Under congested conditions, however, empirical evidence reported by Cassidy and Ahn (2005) indicates that the available capacity on the freeway is allocated to competing streams according to a "merge ratio", as per Daganzo's model (Daganzo 1996), which is a constant independent of flows.

To circumvent this problem, this paper uses a result in Laval and Leclercq (2008) who obtained a continuum model for lane-changing rates in congested traffic. In the case of merges, this means that freeway inflows are expressed as a function of the flow on the freeway and the demand for entering the freeway. The resulting formulation allowed us to better understand the onset of morning and evening commute congestion along crowded freeway corridors, and its relationship with the demand for entering and exiting along the corridor. In order to obtain analytical solutions and better understand system dynamics, a continuum approximation was used to represent inflows and outflows to the corridor.

This paper is organized as follows. Section 2 formulates the problem as a conservation law in both the freeway and the on-ramps, as well as the appropriate boundary conditions. The numerical solution of this problem is carried out in section 3, which reveals considerable insight. Based on this insight, section 4 shows the analytical solution for three important cases. Finally, section 5 presents a discussion and outlook. 


\section{Problem Formulation.}

Consider a long $n$-lane freeway corridor of length $L$ with entrances and exits evenly spaced $\delta$ distance units apart. The number of entrances and exits is large, and therefore freeway inflow and outflow rates can be treated as continuous variables in time $t$ and location $x, \phi^{+}(t, x)$ and $\phi^{-}(t, x)$, in units of veh/time-distance. There exists a homogeneous fundamental diagram $q(k)$ that gives the flow $q(t, x)$ as a function of the local density $k(t, x)$; both quantities defined as totals across all of freeway lanes. Therefore, the traffic conservation law can be expressed as

$$
k_{t}+s(k) k_{x}=\phi^{+}-\phi^{-},
$$

where $s(k)=d q(k) / d k$ is the speed of characteristics, and variables in subscript represent partial derivatives. We will use the superscript " $x$ " to denote variables belonging to the on-ramp that merges at location $x$ in the freeway. With this notation, traffic dynamics at on-ramps are given by

$$
k_{t}^{x}+s^{x}\left(k^{x}\right) k_{y}^{x}=0,
$$

where $k^{x}(t, y)$ gives the density at location $y$ along the on-ramp. The length of all on-ramps is $d$ and are assumed identical to a single freeway lane.

It is reasonable to assume that exit flows are Markovian; i.e.,

$$
\phi^{-}=\beta q,
$$

where $\beta(t, x)$ is the proportion per unit distance of the freeway flow that exits at $(t, x)$. The determination of $\phi^{+}(t, x)$ is more elaborate because one has to capture driver merging behavior, which depends on both the traffic state in the freeway and in the on-ramp. To this end, let $\alpha(t, x)$ be the demand rate for entering the freeway at $(t, x)$, in units of veh/time-distance. Notice that $\alpha$ gives the number of vehicles willing to enter the freeway and not the number of vehicles that actually enter the freeway. To obtain the latter, in this paper we modify eqn. (6) in Laval and Leclercq (2008) which pertains to discretionary lane-changing rates in multilane freeways. Accordingly, the actual freeway inflow rate may be expressed as

$$
\phi^{+}(t, x)=\min \left\{1, \frac{\mu(k(t, x))}{\lambda(k(t, x))}\right\} \lambda^{x}\left(k^{x}(t, d)\right) / \delta,
$$

where $\mu$ and $\lambda$ are the receiving and sending function of kinematic wave theory. For a triangular fundamental diagram with free-flow speed $u$, wave speed $w$, and jam density $\kappa$, we have

$$
\begin{aligned}
\mu(k) & =\min \{(n \kappa-k) w, n Q\}, \\
\lambda(k) & =\min \{u k, n Q\} \\
\mu^{x}\left(k^{x}\right) & =\min \left\{\left(\kappa-k^{x}\right) w, Q\right\} \\
\lambda^{x}\left(k^{x}\right) & =\min \left\{u k^{x}, Q\right\}
\end{aligned}
$$

where $Q=u w \kappa /(u+w)$ is capacity of one lane. Notice that $q(k)=\min \{\lambda(k), \mu(k)\}$ and $q^{x}\left(k^{x}\right)=$ $\min \left\{\lambda^{x}\left(k^{x}\right), \mu^{x}\left(k^{x}\right)\right\}$.

Notice that (4) is the continuum limit (as the cell size goes zero) of the model introduced in Laval and Daganzo (2006) for the flow of lane changes, which allocates the available capacity to each approach according to its sending function. In the case of discrete merges, this approach was first introduced in Jin and Zhang (2003). The appendix shows that this type of models are equivalent to Daganzo's model (Daganzo 1996), which has been validated empirically (Cassidy and Ahn 2005). In particular, they produce constant merging priorities independent of the (congested) flow in the competing approaches. Without loss of generality, (5b) and (5d) imply equal priority between the 
on-ramp and the freeway shoulder lane. The appendix also shows how one can modify (5d) to account for a different priorities.

To model on ramps as a continuum, the term $1 / \delta$ in (4) "diffuses" the demand of a discrete on-ramp (given by (5d)) within a vicinity of length $\delta$. Similarly, we use the following initial and boundary conditions for on-ramps:

$$
\begin{aligned}
k^{x}(0, y) & =\alpha(0, x) \delta / u, \\
k^{x}(t, 0) & =\alpha(t, x) \delta / u, \\
q^{x}(t, d) & =\phi^{+}(t, x) \delta .
\end{aligned}
$$

Initial condition (6a) assumes that all on-ramps are filled with the demand at $t=0$. Boundary conditions (6b) and (6c) imply that the demand at time $t$ enters the on-ramp at $y=0$, and that the flow that actually enters the freeway (i.e., that exits the on-ramp at $y=d$ ) is given by (4).

We also assume that the freeway is empty at $t=0$ and that there is no inflow from the upstream end of the freeway; the only inflow is due to on-ramps in $0 \leq x \leq L$. Notice that the latter is without loss of generality because one can always increase $L$ to account for higher freeway flows at a given location. Thus, in addition to (3) and (4), the following are the initial and boundary conditions for the freeway:

$$
\begin{aligned}
k(0, x) & =0, \\
k(t, 0) & =0 .
\end{aligned}
$$

As formulated, the problem is general enough to capture any demand pattern whatsoever. The numerical solutions for such problems would be straightforward, but simulation is a lousy tool for understanding the problem. Therefore, in the sequel we restrict our attention to time-independent and location-monotonic demand patterns, i.e., $\alpha=\alpha(x), \beta=\beta(x), d^{2} \alpha / d x^{2}=d^{2} \beta / d x^{2}=0$. Depending on the sign of the slope of $\alpha(x)$ and $\beta(x)$, this should provide an adequate approximation for the morning and evening commute problems. Additionally, this simplification will allow us to obtain analytical solutions that reveal important insights which should be true in general.

The next section shows the numerical solution of the problem formulated in this section. The numerical solution reveals important properties of the problem, which are useful for the analytical solution presented in section 4 .

\section{Numerical Solution.}

In this section we apply Godunov's scheme to the problem (1)-(7) solving the Riemann problems using the sending and receiving functions (5) as described in Daganzo (1996). Without loss of generality, we have chosen $w=u$ in these experiments in order to obtain exact solutions, i.e., free of numerical errors (the reader is referred to Leclercq et al. 2007, for a demonstration). For the numerical examples in this section we have used $n=3$ lanes, $w=u=100 \mathrm{~km} / \mathrm{hr}$ and $\kappa=150 \mathrm{veh} / \mathrm{km}$ (and therefore $Q=7500 \mathrm{veh} / \mathrm{hr}$ ).

The main insight revealed by the numerical solutions is that there exists four distinct regions in the time-space plane where density obeys a distinctive pattern: two of these regions correspond to free-flow states (labeled regions $\mathrm{A}$ and $\mathrm{B}$ in the sequel) and two are congested (C and D). Regions $\mathrm{A}$ and $\mathrm{C}$ are transient while $\mathrm{B}$ and $\mathrm{D}$ give the steady state of the system (until demands drop at the end of the rush hour). Of course, the evolution of density inside each region will depend on the functions $\alpha(x)$ and $\beta(x)$, but as we will see in the sequel the shape of the regions is rather general for typical rush-hour patterns. 
(a)

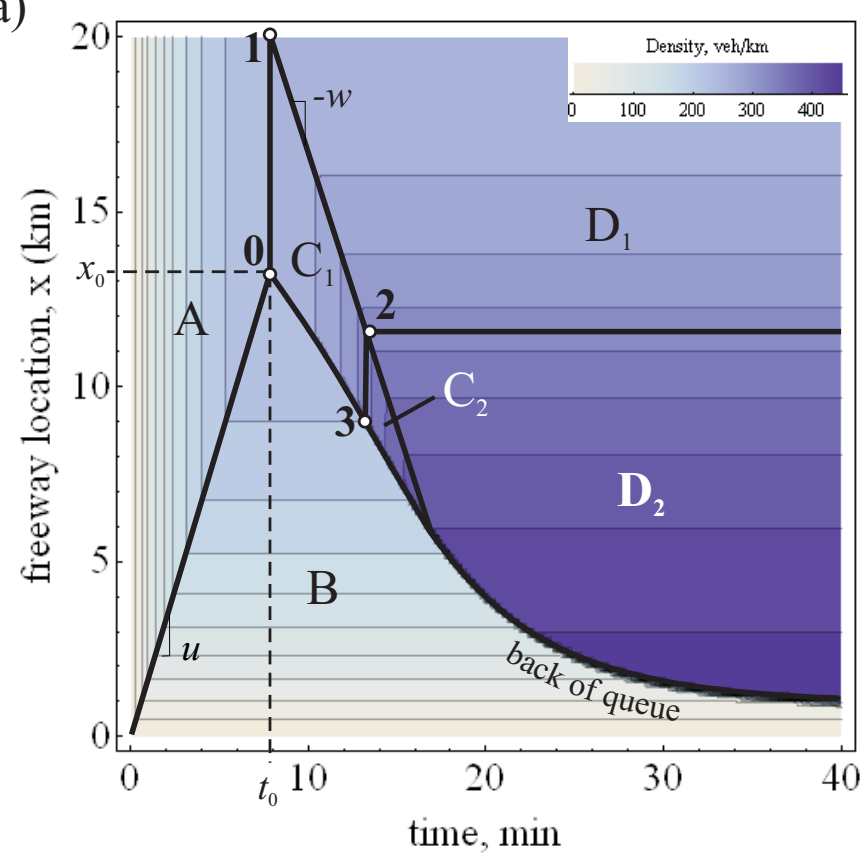

(b)
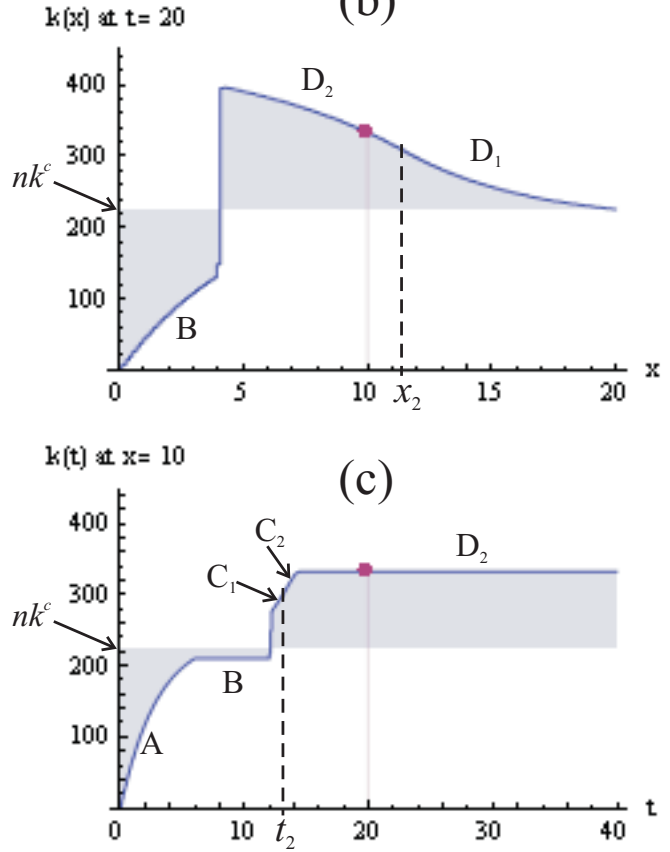

Figure 1 Numerical solution for the constant demand case: $a=4,850 \mathbf{v e h} / \mathbf{h r} / \mathbf{k m}, b=0.2 \mathbf{k m}^{-1}$ and $L=20 \mathbf{k m}$ and $\delta=1 \mathrm{~km}$. (a) Time-space density contour map for the freeway. Bold lines correspond to interfaces between the different traffic states, while thin lines are isodensity contours. (b) and (c): evolution of density at $t=20 \mathrm{~min}$ and $x=10 \mathrm{~km}$, respectively.

\subsection{Constant demands}

We start our discussion with the simplest case where $\alpha(x)=a$ and $\beta(x)=b$ are constant in $0<$ $x<L$ and zero elsewhere. Figure 1a shows a density contour map obtained numerically. Region A corresponds to the "filling up" of the freeway, where, as time passes, the density increases. As can be seen in figure the isodensity contours are vertical, meaning that at a given time the density in the freeway is constant for $x \geq u t$. Inside region B densities have reached a free-flow equilibrium, where isodensity contours are horizontal. Point " 0 " in the figure marks the beginning of congestion. At this point the density reaches the critical density and therefore a shock waves propagates upstream, which corresponds to the back of the queue. At the same time, a wave is emanated from point "1" in the figure, which is not a result of a restriction from downstream of $x=L$ but of the absence of lateral inflow and outflow for $x>L$. This wave eventually meets the back of the queue, and marks the boundary between congested regions $\mathrm{C}$ and $\mathrm{D}$. The difference between these regions is that in the former the isodensity contours are vertical and in the latter, horizontal. These congested regions can be broken down into two subregions: regions $\mathrm{C}_{1}$ and $\mathrm{D}_{1}$ where on-ramps are in free-flow conditions, and regions $\mathrm{C}_{2}$ and $\mathrm{D}_{2}$ where on-ramps are congested. The boundary between the two sub regions are defined by points "2" and " 3 " in the figure. Point "2" corresponds to the most downstream location where on-ramp congestion is first observed, while point " 3 " is the analogous for the most upstream location.

Figures Fig. 1b and 1c show the evolution of density at $t=20 \mathrm{~min}$ and $x=10 \mathrm{~km}$, respectively. As expected, there is a sharp shock between region $\mathrm{B}$ and the congested regions. It can be seen in part b of the figure that point " 2 " corresponds to an inflection point in the spatial evolution of density inside region $\mathrm{D}$.

A density map at selected time instants is presented in Fig. 2 for both the freeway and the onramps. Consistently with Fig. 1a, it can be seen that congestion at on-ramps starts around $t=14$ min simultaneously between locations $x_{2}$ and $x_{3}$. On-ramp queues propagate upstream thereafter, 


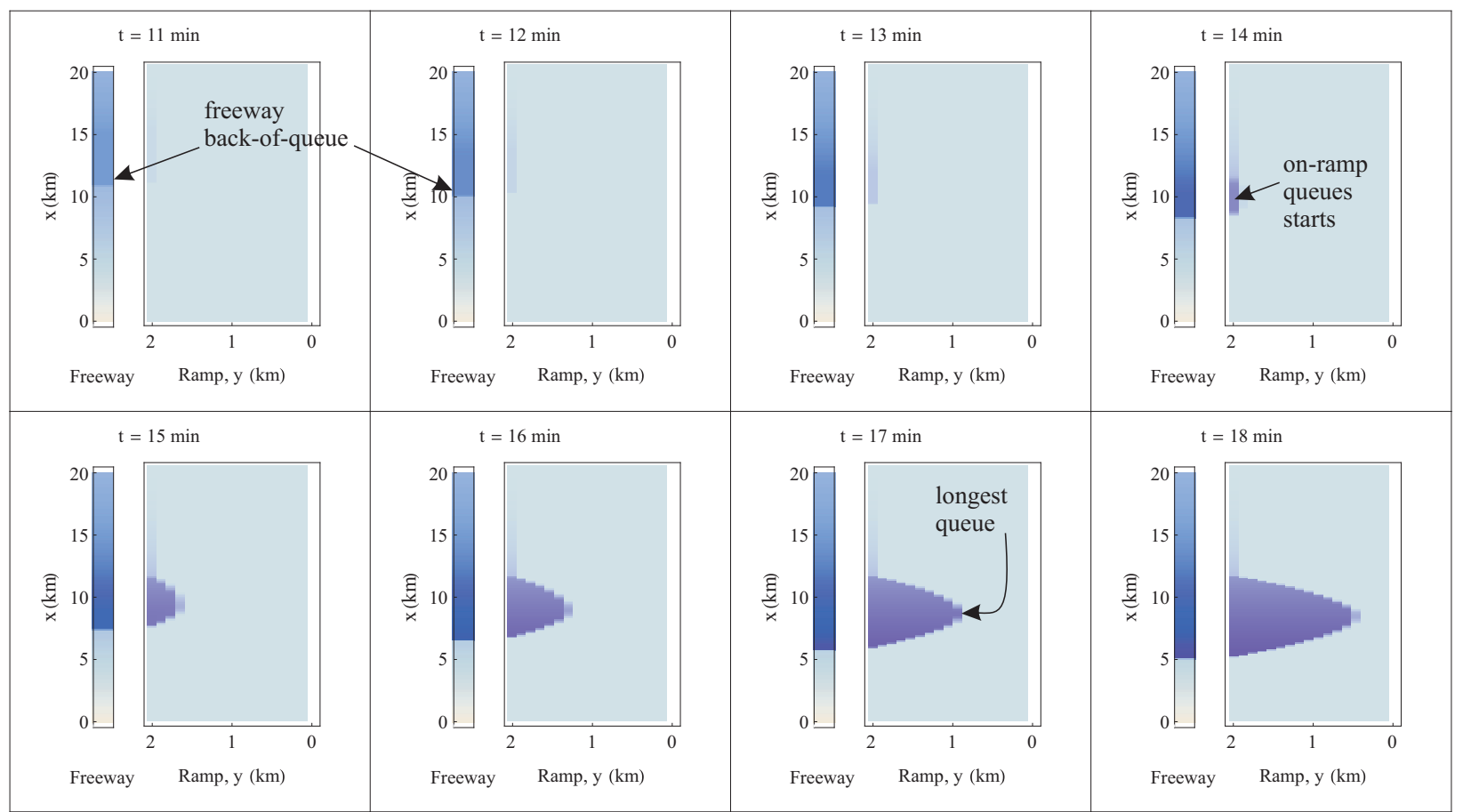

Figure 2 A density map at selected time instants for both the freeway and the on-ramps.

eventually reaching the beginning of the on-ramp at $y=0$. It is interesting to note that at a given time instant larger queues are observed roughly in the midpoint between $x_{2}$ and the back of the freeway queue.

\subsection{Monotonic demand patterns.}

Here we extend our previous analysis to include a first-order dependency between the demand and freeway location. To this end, we consider all combinations, the following specifications for on-ramps:

$$
\begin{aligned}
& \alpha(x)=a, \\
& \alpha(x)=a(1-x / L), \\
& \alpha(x)=a x / L
\end{aligned}
$$

(constant demand)

(decreasing demand)

(increasing demand)

and the for exits:

$$
\begin{aligned}
& \beta(x)=b, \\
& \beta(x)=b(1-x / L), \\
& \beta(x)=b x / L,
\end{aligned}
$$

(constant demand)

(decreasing demand)

(increasing demand)

Notice that specifications (8) and (9) are valid in $0 \leq x \leq L$; elsewhere all functions are identically zero.

The numerical solution for all combinations between (8) and (9) are shown in Fig. 3. It can be seen that the main qualitative difference with respect to the constant demand case of Fig. 1 are that

1. the isodensity contours in regions $\mathrm{A}$ and $\mathrm{C}$ are no longer vertical, and are determined by on-ramp demand. This can be verified by comparing the second and third row of the figure.

2. there is a new free-flow region $B_{2}$ that appears downstream of $D_{1}$ in three cases(parts c, $d$ and $\mathrm{f}$ in the figure). These regions appear because of the small on-ramp demand and-or a large exit demand near the end of the freeway segment. 


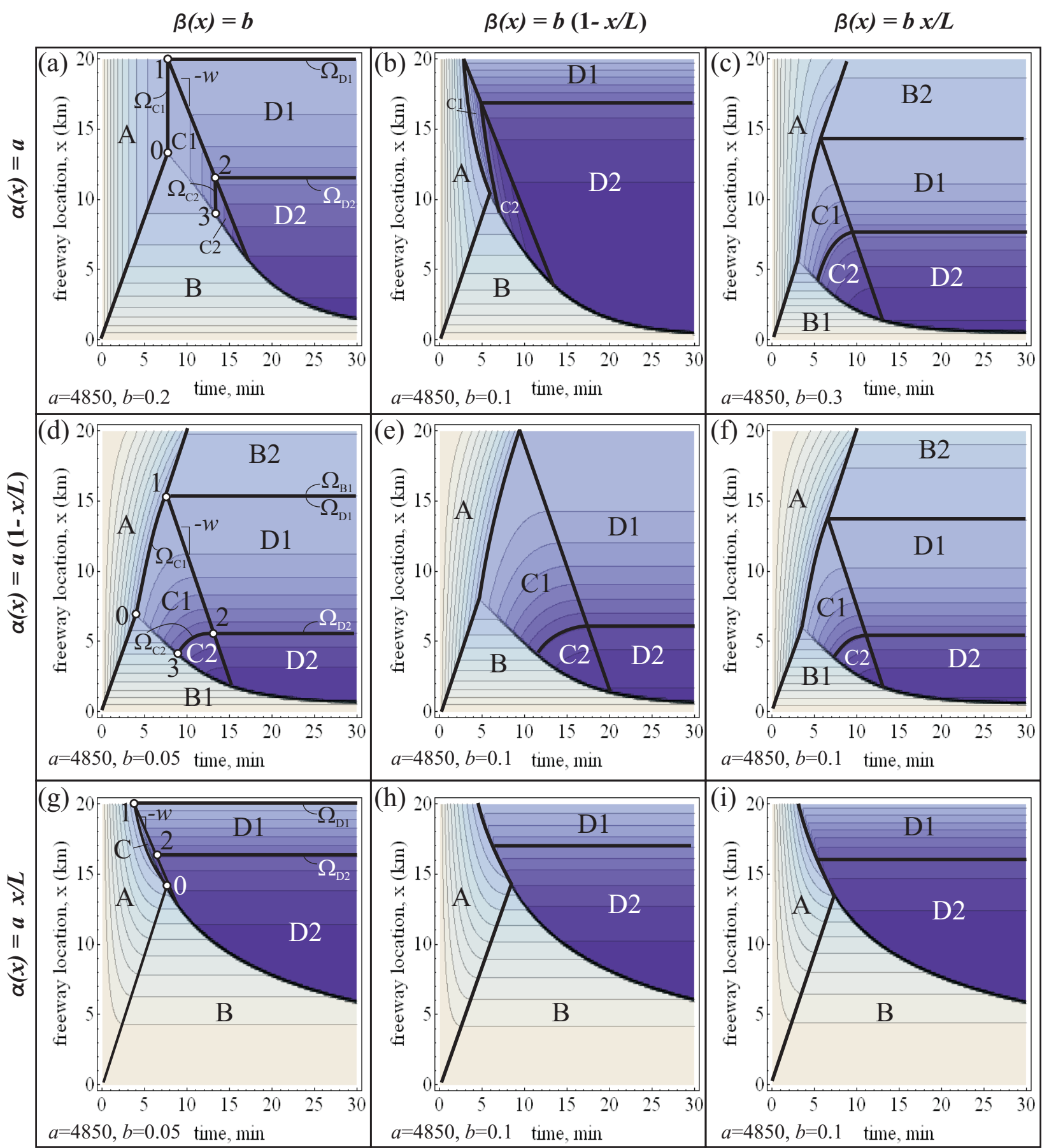

Figure 3 Numerical solution for all combinations between (8) and (9). Bold lines correspond to interfaces between the different traffic states, while thin lines are isodensity contours.

3. region $\mathrm{C}$ tends to disappear (but still exists) in the case of increasing on-ramp demand; see last row in the figure.

4. the interface between regions $\mathrm{C}$ and $\mathrm{A}$ are either forward moving (parts $\mathrm{c}, \mathrm{d}$, e and $\mathrm{f}$ of the figure) or backward moving (b, g, h and i).

Notice how pictures $\mathrm{d}$ and $\mathrm{g}$ in the figure are similar to all cases within their respective row. This suggests that, at least qualitatively, the behavior of the system is not very sensitive to the exit demand pattern, and that the constant exit demand case should provide good approximations for 
more complicated patterns. A similar argument can be made for the constant on-ramp demand. It is apparent how pictures $\mathrm{b}$ and $\mathrm{c}$ in the figure are very similar to the pictures in the third and second row, respectively. This result is intuitive since, in terms of net inflow, an increasing (decreasing) exit demand and a decreasing (increasing) on demand are interchangeable.

As expected, congestion is more severe farther away from the downtown area, in all cases. This is evident from the figure since higher densities are observed closer to the beginning of the freeway segment.

\section{Analytical Solution}

In this section we derive the analytical solution for the three cases in the first column of Fig. (3), i.e. $\beta(x)=b$. Unfortunately, these are the only cases where one can obtain fully algebraic solutions; all other cases involve terms that can only be evaluated numerically. However, it was founded in section 3 that the particular form of $\beta(x)$ does not affect significantly the results compared to the constant- $\beta$ case, at least qualitatively. Therefore, it is expected that the insights obtained for the three cases analyzed in this section are valid in general.

The solution method proposed here recognizes that inside each region identified in the previous section, the density obeys a simplified form of (1) supplemented with "ad-hoc" boundary conditions. In particular, since each region is either congested or uncongested, the speed of characteristics $s(k)$ is either $-w$ or $u$, respectively, independent of the density, which facilitates matters considerably. The idea then is to solve each region independently choosing the appropriate boundary conditions.

In general, a boundary condition specifies (exogenous) values for the density, $k_{0}(t, x)$, along a trajectory in the time-space plane, $(t, x) \in \Omega$; i.e.:

$$
k(t, x)=k_{0}(t, x), \quad \forall(t, x) \in \Omega .
$$

where $\Omega$ is the set of points defining the trajectory. For the examples of the previous section, part a of Fig. 3 shows the trajectory relevant for each region, $\Omega_{\mathrm{A}}, \Omega_{\mathrm{B}} \ldots \Omega_{\mathrm{D}_{2}}$.

It is important to note that the proposed region-by-region solution method does not require the explicit solution of the kinematic wave model for on-ramps (2). This is true because inside each region the inflow rate $\phi^{+}$can be determined exogenously. In particular, $\phi^{+}$takes only two possible values depending on the traffic states prevailing in the on-ramp just upstream of the merge; i.e.:

$$
\phi^{+}(t, x)=\left\{\begin{array}{lr}
\alpha(x), & \text { (uncongested on-ramp) }, \\
(n \kappa-k(t, x)) w /(n \delta), & \text { (congested on-ramp). }
\end{array}\right.
$$

It is clear that in free-flow $\phi^{+}$corresponds to the on-ramp demand $\alpha(x)$. To obtain the congested portion of (11), we note that on-ramp congestion takes place only when the freeway is congested, in which case (4) can be written as $\phi^{+}=\mu \lambda^{x} /(\lambda \delta)$. Since both the freeway and the on-ramp are congested then $\mu=(n \kappa-k) w, \lambda=n Q$ and $\lambda^{x}=Q$, and therefore $\phi^{+}=(n \kappa-k) w /(n \delta)$ as sought.

Finally, Table 1 shows a summary of all the information needed to solve the kinematic wave model (1) inside each region, for the case of constant demands. All other cases are solved similarly, the only difference being the definition of the boundary conditions. Note that in the table $k^{c}$ refers to the critical density in a single lane.

\subsection{Constant demands}

In this section we consider demands that are constant along the freeway corridor; as per (8b) and (9a). Next, we use Table 1 to obtain the analytical expressions for the density inside regions A 
Table 1 Summary of specifications for solving the kinematic wave model (1)-(7) inside each region

\begin{tabular}{ccccccc}
\hline region & freeway & on-ramps & $s(k)$ & $\phi^{+}-\phi^{-}$ & $\Omega$ & $k_{0}(t, x)$ \\
\hline $\mathrm{A}$ & free-flow & free-flow & $u$ & $\alpha-\beta u k$ & $t=0$ & 0 \\
$\mathrm{~B}$ & free-flow & free-flow & $u$ & $\alpha-\beta u k$ & $x=0$ & 0 \\
$\mathrm{C} 1$ & congested & free-flow & $-w$ & $\alpha-\beta(n \kappa-k) w$ & $t=t_{0}$ & $n k^{c}$ \\
$\mathrm{C} 2$ & congested & congested & $-w$ & $(1 /(n \delta)-\beta)(n \kappa-k) w$ & $t=t_{2}$ & $k_{C_{1}}\left(t_{2}, \Omega_{\mathrm{C}_{2}}\right)$ \\
$\mathrm{D} 1$ & congested & free-flow & $-w$ & $\alpha-\beta(n \kappa-k) w$ & $x=x_{1}$ & $n k^{c}$ \\
$\mathrm{D} 2$ & congested & congested & $-w$ & $(1 /(n \delta)-\beta)(n \kappa-k) w$ & $x=x_{2}$ & $k_{D_{1}}\left(t_{2}, \Omega_{\mathrm{D}_{2}}\right)$ \\
\hline
\end{tabular}

through $\mathrm{D}_{2}$, which are labeled $k_{A}, k_{B} \ldots$. This can be accomplished using the method of characteristics(see e.g. LeVeque 1993), which recognizes that along characteristic lines the density obeys an ordinary differential equation that can be solved straightforwardly. It can be shown that:

$$
\begin{aligned}
k_{A}(t) & =(1-\exp (-b u t)) a /(u b), \\
k_{B}(x) & =(1-\exp (-b x)) a /(u b), \\
k_{C 1}(t) & =n \kappa-\frac{1}{b w}\left(a\left(1-c_{1}^{\frac{w}{u}+1}\right) \exp (b w t)\right), \\
k_{C 2}(t) & =n \kappa-\frac{n \delta a}{w}\left(\frac{c_{0}}{c_{1}}\right)^{\frac{1}{b \delta n}-1}\left(\frac{1}{c_{1}}\right)^{\frac{c_{0} w}{b \delta n u}} \exp \left(-\frac{w c_{0}}{\delta n} t\right), \\
k_{D 1}(x) & =n \kappa-\frac{1}{b w}\left(a\left(1+c_{1}\right) \exp (b(L-x))\right), \\
k_{D 2}(x) & =n \kappa-\frac{n \delta a}{w}\left(\frac{c_{0}}{c_{1}}\right)^{\frac{1}{b \delta n}-1} \exp \left(-\frac{c_{0}}{\delta n}(L-x)\right) .
\end{aligned}
$$

where we have defined the (dimensionless) constants

$$
\begin{aligned}
& c_{0}=1-b n \delta, \\
& c_{1}=1-b n Q / a .
\end{aligned}
$$

The above solutions are valid only in the relevant regions $A \ldots D_{2}$ defined in Fig. 1 . To formalize the definition of these regions we will next find the coordinates $\left(t_{i}, x_{i}\right)$ of points $i=0,1,2,3$ in the figure.

Point " 0 " is the point closest to the origin where the density equals the critical density. Therefore, it can be obtained by solving $k_{B}(x)=n k^{c}$ for $x$, which gives

$$
\begin{aligned}
x_{0} & =\frac{1}{b} \log \left(\frac{1}{c_{1}}\right), \\
t_{0} & =x_{0} / u .
\end{aligned}
$$

In the numerical example of Fig. 1 this gives $x_{0}=13.14 \mathrm{~km}$ and $t_{0}=7.88 \mathrm{~min}$, as can be verified in the figure. Clearly,

$$
\begin{gathered}
x_{1}=L, \\
t_{1}=t_{0} .
\end{gathered}
$$

The location of point " 2 ", $x_{2}$, marks the location of the most downstream on-ramp that first suffers congestion. At this point the demand on the on-ramp $a \delta$ equals the capacity allocated to the onramp according to the merge model, $q\left(k_{D 1}(x)\right) / n$. Accordingly, solving $a \delta=\left(n \kappa-k_{D 1}(x)\right) w / n$ for $x$ gives

$$
\begin{aligned}
x_{2} & =L-\frac{1}{b} \log \left(\frac{c_{0}}{c_{1}}\right), \quad \text { and } \\
t_{2} & =\left(L-x_{2}\right) / w+t_{0},
\end{aligned}
$$


which gives $x_{2}=11.44$ and $t_{2}=13.02$ min for Fig. 1 .

The formulae for points " 0 " and "2" above can be used to obtain the conditions for the existence of congestion both in the freeway and the on-ramps. In particular, no freeway congestion means that point "0" appears beyond the length of our freeway segment; i.e., $x_{0}>L$. Similarly no congestion on the on-ramps means $x_{2}<0$. Using (14a) and (16a) gives

$$
\begin{aligned}
& a \leq \frac{b n Q}{1-e^{-b L}}, \quad \text { (no freeway congestion) } \\
& a \leq \frac{b n Q}{1-c_{0} e^{-L b}} . \quad \text { (no on-ramp congestion) }
\end{aligned}
$$

In the numerical example of Fig. 1 we obtained the conditions $a \leq 4,533.2$ and $a \leq 4,584$, respectively. Notice that for very long freeway segments the exponential terms in (17) vanish, so that a common no-congestion condition simplifies to $a \leq b n Q$.

As was mentioned in section 3 , the highest densities are observed near the beginning of the freeway segment, i.e., in region $\mathrm{D}_{2}$. But this high density is always smaller than the jam density. In fact, the limit of (12f) tends to the jam density only for very long freeways; i.e., as $L \rightarrow \infty$. For example, in the case of Fig. 1 we have that for $L=20 \mathrm{~km} k_{D_{2}}(0)=418<n \kappa=450 \mathrm{veh} / \mathrm{km}$.

It is possible to obtain the equation of the back of the queue; see Fig. 1a. This can be done using the classic "shock condition" which asserts that the speed of the shock is given by the ratio between the difference in flow and the difference density between the neighboring traffic states, respectively. In our case, one would obtain three formulae for the shock speed, one for each pair of neighboring traffic states $\mathrm{B}-\mathrm{C}_{1}, \mathrm{~B}-\mathrm{C}_{2}$ and $\mathrm{B}-\mathrm{D}_{2}$. Unfortunately, these equations are very complicated and not particularly insightful and therefore are not included here.

\subsection{Decreasing on-ramp demand.}

In this section we assume demands obeying (8b) and (9a); i.e., on-ramp demand decreases linearly along the freeway. This could represent a first-order approximation for morning (evening) commute in cities where people tend to live in the suburbs (downtown area). The relevant boundary conditions are shown in Fig. 3d. Notice that these are similar to the constant demand case in Table 1 except for state $\mathrm{C}$ and the new state $\mathrm{B}_{2}$. In the latter case the boundary condition is simply $k\left(t, x_{1}\right)=n k^{c}$, but unfortunately for $\mathrm{C}_{1}$ and $\mathrm{C}_{2}$ the boundary conditions are far more complicated. In fact, as can be seen in the figure, $\Omega_{\mathrm{C}_{1}}(t)$ and $\Omega_{\mathrm{C}_{2}}(t)$ correspond to the isodensity curves $k_{A}(t, x)=n k^{c}$ and $k_{C_{1}}(t, x)=k_{D_{1}}\left(x_{2}\right)$, respectively, which turn the problem mathematically intractable. This has no profound impact in our analysis since these regions are only transient. For the remaining regions it can be shown that

$$
\begin{aligned}
k_{A}(t, x) & =c_{4}\left(1+b(L-x)-e^{-b t u}(b(L+t u-x)-1)\right), \\
k_{B_{1}}(x) & =c_{4}\left(1+b(L-x)-e^{-b x}(b L+1)\right), \\
k_{B_{2}}(x) & =c_{4}\left(1+b(L-x)-e^{-b\left(c_{2}-L+x\right)}\right), \\
k_{D_{1}}(x) & =n \kappa-c_{4}\left(1+b(L-x)-e^{-b\left(c_{2}-L+x\right)}\right), \\
k_{D_{2}}(x) & =n \kappa-c_{4} e^{-b c_{2}-\frac{L-x c_{0}-\frac{c_{3}}{b}}{\delta n}}\left(e^{b\left(c_{2}+L\right)-c_{3}}\left(1+c_{3}\right)-e^{b L}\right),
\end{aligned}
$$

where we have defined the (positive) constants

$$
\begin{aligned}
c_{2} & =n Q L b / a, \\
c_{3} & =-\mathcal{W}\left(-\frac{1}{c_{0}} \exp \left(-b c_{2}-\frac{1}{c_{0}}\right)\right)-\frac{1}{c_{0}}, \\
c_{4} & =\frac{a}{b^{2} L u} .
\end{aligned}
$$


In $(19 b)$ the term $\mathcal{W}(z)$ represent the (real-valued) Lambert $W$-function, which gives the solution of $z=w e^{w}$. The coordinates for the different points in Fig. 3d can be obtained similarly to the constant demand case. Solving $k_{B_{1}}(x)=n k^{c}$ for $x$ gives

$$
\begin{aligned}
x_{0} & =L-c_{2}+\frac{1}{b}\left(1+\mathcal{W}\left(-(b L+1) \exp \left(-b\left(L-c_{2}\right)-1\right)\right)\right), \\
t_{0} & =x_{0} / u
\end{aligned}
$$

For the example in Fig. $3 \mathrm{~d}$ we have that $x_{0}=7.48 \mathrm{~km}$ and $t_{0}=x_{0} / u=4.48 \mathrm{~min}$. To obtain point " 1 " we note that it is the most downstream point in A where the density equals $n k^{c}$. It is straightforward to show that

$$
\begin{aligned}
x_{1} & =L-\left(e^{b t u}-1\right) / b+u t_{1}, \\
t_{1} & =-c_{2} / u-\left(1+\mathcal{W}\left(-\exp \left(-b c_{2}-1\right)\right)\right) /(b u),
\end{aligned}
$$

which in the case of Fig. $3 \mathrm{~d}$ gives $t_{1}=7.59 \mathrm{~min}$ and $x_{1}=15 \mathrm{~km}$. Solving $\delta \alpha(x)=w\left(n \kappa-k_{D 1}(x)\right) / n$ for $x$ gives

$$
\begin{aligned}
x_{2} & =L-c_{3} / b, \\
t_{2} & =\left(x_{1}-x_{2}\right) / w+t_{1} .
\end{aligned}
$$

In the case of Fig. $3 \mathrm{~d}$ this gives $t_{2}=13.42 \mathrm{~min}$ and $x_{2}=5.28 \mathrm{~km}$. With all, the congestion conditions become

$$
\begin{array}{lr}
a \leq \frac{b^{2} n Q L}{1-(b L+1) \exp (-b L)}, & \text { (no freeway congestion) } \\
a \leq \frac{b^{2} n Q L}{b L-\log \left(b L c_{0}+1\right)} . & \text { (no on-ramp congestion) }
\end{array}
$$

which for Fig. 3d give $a \leq 4,257.5$ and $a \leq 2,923.5$, respectively. Interestingly, for very long freeways one obtains the same non-congestion condition $a \leq b n Q$ as in the constant demand case. The reader can verify this by taking the limit $L \rightarrow \infty$ in (23).

Similarly to the constant on-ramp demand case the limit of (18e) tends to the jam density as $L \rightarrow \infty$. For Fig. $3 d$ we have $k_{D_{2}}(0)=431<n \kappa=450 \mathrm{veh} / \mathrm{km}$.

\subsection{Increasing on-ramp demand}

Here demands are given by (8c) and (9a); i.e., on-ramp demand decreases linearly along the freeway, as an approximation for the morning (evening) commute cities where people tend to live in the downtown area (suburbs). Proceeding similarly as in previous sections, a summary of results follow.

$$
\begin{aligned}
k_{A}(t, x) & =c_{4}\left(b u t+(1-b x)\left(1-e^{b t u}\right)\right), \\
k_{B}(x) & =c_{4}\left(b x-1+e^{-b x}\right) \\
k_{D 1}(x) & =n \kappa-c_{4}\left(1+b(L-x)-e^{-b\left(c_{2}-L+x\right)}\right), \\
k_{D 2}(x) & =n \kappa-c_{4}\left(e^{b L-\frac{c_{5}}{b \delta n}+\frac{x c_{0}}{\delta n}}\left(1-b\left(L-c_{2}\right)\right)-e^{-\frac{\left(c_{5}-b x\right) c_{0}+1}{b \delta n}}\left(1-c_{5}\right)\right),
\end{aligned}
$$

where $c_{5}=\mathcal{W}\left(-\frac{1}{c_{0}} e^{b L-\frac{1}{c_{0}}}\left(1-b\left(L-c_{2}\right)\right)\right)+\frac{1}{c_{0}}$ is a dimensionless constant. As opposed to the previous two sections, the back of the queue starts in point "1" in Fig. $3 \mathrm{~g}$, which is located at the downstream end of the freeway segment. The time when congestion starts, $t_{1}$, is such that $k_{A}\left(t_{1}, L\right)=n k^{c} ;$ i.e.:

$$
\begin{aligned}
x_{1} & =L, \\
t_{1} & =\frac{1}{b u}\left(b L-1-\mathcal{W}\left(e^{b L-1}\left(b L-1-c_{2}\right)\right)\right)
\end{aligned}
$$


which in the case of Fig. $3 \mathrm{~g}$ gives $t_{1}=3.83$ min. Point "2" is given by

$$
\begin{aligned}
x_{2} & =c_{5} / b, \\
t_{2} & =\left(L-x_{2}\right) / w+t_{1} .
\end{aligned}
$$

Unfortunately, point " 0 " is located in the back of the queue, and it becomes mathematically intractable to obtain its coordinates. However, we can still find the congested conditions. In the case of the freeway, this condition is equivalent to having $t_{1} \geq L / u$, since $L / u$ is the latest time for congestion to form (after this time the freeway is in steady state). In the case of the on-ramps, we can use the same argument as in previous sections, i.e., $x_{2}<0$. Interestingly, these two conditions are mathematically equivalent; i.e.:

$$
a \leq \frac{b^{2} n Q L}{b L-1+\exp (-b L)}, \quad \quad \text { (no freeway and on-ramp congestion) }
$$

which also tends to $a \leq b n Q$ for very long freeways.

\section{Discussion.}

The continuum approximation proposed in this paper reveals considerable insights on the operation of freeway corridors, and yet it requires only two parameters, $a$ and $b$. As in any continuum approximation, one has to interpret the results with care when comparing with real-world situations. For example, one should interpret vehicular density as an indication of the likelihood to encounter congestion at a given time-space point. It is clear that in the real world demands are far more complicated than idealized here, yet our results give a first order approximation. And even if real-world demands behaved as postulated here, the accuracy of the predictions would decrease with the actual distance between ramps, $\delta$.

Of particular interest is region $\mathrm{C}$, which is a transient and marks the beginning of congestion. The on-ramps covered by this region (e.g., the ones located, roughly, in $x_{0} \leq x \leq x_{1}$ ) should be interpreted as the most likely on-ramps to become active bottlenecks. In fact, it is commonly observed in the field that the location of the active bottleneck in a freeway corridor varies from day to day, but always within a relatively short segment. This can be seen in Fig. 4, which presents a collection of density maps for 12 days from a real freeway corridor. It can be seen how the active bottleneck changes location within the same rush hour following a rather reproducible pattern in time-space. This is consistent with the shape of the interface between regions $\mathrm{C}$ and $\mathrm{A}$ shown in Fig. 3, which can be either forward moving (parts c, d, e and $\mathrm{f}$ of the figure), backward moving (b, g, h and i) or vertical (a). In each case one would expect that the active bottleneck would shift location according to the shape of this interface.

The findings in this paper may be used to explore a new kind of ramp metering strategy. It was found that on-ramp queues form in a well-defined portion of the freeway segment, i.e., in $x \leq x_{2}$. Since on-ramp queues can be very deleterious (capacity drop and spill-back to city streets) one can devise a ramp metering strategy that would spread on-ramp queues more evenly across the entire freeway corridor. This could be done by metering the on-ramps downstream of $x_{2}$ in such a way that the freeway flow in $x \leq x_{2}$ enables higher on-ramp discharge. Notice that this result is independent of the demand profile. While other authors have pointed out that for minimizing total cost the closer to the CBD the more restrictive metering rates should be, our finding is different: (i) there may be a multitude of strategies (but always implemented downstream of $x_{2}$ ) that may achieve similar queue lengths distribution; and (ii) Figs. 3c and 3f show that there are instances where there is no reason for metering near the CBD as the freeway there is in free-flow conditions. Research in this realm continues. 


\section{Appendix A: The Merge Model.}

This appendix shows that (4) is equivalent to Daganzo's model (Daganzo 1996), and that one needs a single additional parameter, $\zeta$, to modify (5d) in order to obtain experimentally observed merge ratios (Cassidy and Ahn 2005). To simplify the exposition we consider a "discrete" merge for the demonstration in the first subsection, and show how to obtain the parameter $\zeta$ in the continuum approximation in the second subsection.

\section{A.1. Discrete on-ramps.}

Consider Fig. 5 which shows a discrete on-ramp and its "merge diagram" describing the actual flows through the merge, $q_{i}$ in approach $i=1$ (freeway), 2 (on-ramp), given a demand vector $\left(q_{1}^{0}, q_{2}^{0}\right)$. A demand vector is feasible if each component is less than the approach capacity $Q_{i}$, and if it is below the total capacity line in the figure; i.e., if the total demand $q_{1}+q_{2}$ is less than the available capacity downstream of the merge, $\mu_{1}$. The merge ratio $p$ is such that when both approaches are congested, each one discharges at a "minimum capacity" $q_{i}^{*}, i=1,2$; i.e., $p=q_{2}^{*} / q_{1}^{*}$ and $q_{1}^{*}=\mu_{1} /(1+p), q_{2}^{*}=\mu_{1} p /(1+p)$. An approach is said to the congested if $q_{i}^{0}>q_{i}^{*}$. Using this notation (4) can be expressed as

$$
\begin{aligned}
& q_{2}=\min \left\{1, \frac{\mu_{1}}{\lambda_{1}}\right\} \lambda_{2}, \quad \text { while } \\
& q_{1}=\mu_{1}-q_{2},
\end{aligned}
$$

where $\lambda_{i}$ is the sending functions for approach $i=1,2$. Using $Q_{1}=n Q$ and $Q_{2}=\zeta Q$ these demands can be expressed as

$$
\begin{aligned}
& \lambda_{1}=\min \left\{q_{1}^{0}, n Q\right\}, \\
& \lambda_{2}=\min \left\{q_{2}^{0}, \zeta Q\right\} .
\end{aligned}
$$

where we have introduced the parameter $\zeta$ for allowing calibrating the model against empirical merge ratios.

To prove that (4) is equivalent to Daganzo's model, we note that a merge can be in one of three states: both approach in free-flow, both approaches in congestion, and one approach congested and the other one in free-flow. Next we examine each case individually.

When all approaches are in free-flow $\mu_{1} \geq \lambda_{1}$ and $\lambda_{i}=q_{i}^{0}, i=1,2$ so that (28)-(29) give $q_{2}=q_{2}^{0}$, as expected. When all approaches are congested $\mu_{1} \leq \lambda_{1}=n Q$ and $\lambda_{2}=\zeta Q$ and therefore $q_{2}=\mu_{1} \zeta / n$. Since in this case $q_{2}$ should equal the prediction of Daganzo's model $q_{2}^{*}=\mu_{1} p /(1+p)$, it is clear that one should choose $\zeta$ as

$$
\zeta=\frac{n p}{1+p} .
$$

Now we examine the case where the freeway is congested and the on-ramp is uncongested. In this case one gets $q_{2}=\mu_{1} q_{2}^{0} /(n Q)$, which corresponds to point "2" in Fig. 5a. But this point is not stable because on-ramp demand is greater than the allocated capacity, i.e. $q_{2}^{0} \geq \mu_{1} q_{2}^{0} /(n Q)$. Therefore, a queue will grow in the on-ramp propagating upstream at a speed $s_{2}$; see part b of the figure. However, as soon as the queue appears in the on-ramp, the sending function $\lambda_{2}$ jumps to its maximum value $\zeta Q$, bringing total demand to point "3" in Fig. 5a. At this point, (28)-(29) give $q_{2}=\mu_{1} \zeta / n$; i.e., point "4" in the figure. Again, this point is not stable because $q_{2}^{0} \leq \mu_{1} \zeta / n$, which means that the on-ramp back-of-queue will eventually recede at a speed $s_{4}$ in Fig. $5 \mathrm{~b}$. As soon as this queue clears, total demand will be given by point "0" 'and the cycle starts over. This cycle produces a "flip-flop" between points "2" and "4" in the figure. It turns out that the long-run average between these points is point " 1 ", which establishes the sought equivalence. This is true because the on-ramp queue does not grow indefinitely, but goes back and forth at speeds $s_{2}$ and $s_{4}$, respectively. This means that the long-run average flow discharging from the on-ramp equals its input flow, $q_{2}=q_{2}^{0}$. Clearly, $q_{1}=\mu_{1}-q_{2}$. This concludes the demonstration.

\section{A.2. Continuous on-ramps.}

Here we show how to choose $\zeta$ in the continuum approximation. Consider a single on-ramp at $x=0$ and let $x=\delta$ be the beginning of the next upstream on-ramp. Using the notation in the main text, it is now clear that when both the freeway and the on-ramp are congested then $\phi^{+}(x)=q(x) \zeta /(n \delta), 0 \leq x \leq \delta$. In steady-state conditions the conservation equation can be expressed as the ODE:

$$
\begin{aligned}
\frac{d q(x)}{d x} & =q(x) \frac{\zeta}{n \delta}, \\
q(0) & =\mu_{1},
\end{aligned}
$$


whose solution is $q(x)=\mu_{1} \exp (-x \zeta /(n \delta))$. Evaluating this solution at $x=\delta$ and combining it with Daganzo's model prediction, i.e. $q(\delta)=\mu_{1} /(1+p)$, gives the appropriate value for $\zeta$,

$$
\zeta=n \ln (1+p) .
$$

Finally, it is worth noting that for simulation purposes, it is straightforward to incorporate explicitly the lengths, $\Delta$, of the insertion section (i.e., the freeway section where vehicles can change lanes from the onramp to the freeway). The only changes are (i) replacing $\delta$ with $\Delta$, and (ii) set $\phi^{+}=0$ outside the insertion sections.

\section{References}

Bastin, G, B Haut, J Coron, B d'Andra Novel. 2007. Lyapunov stability analysis of networks of scalar conservation laws. Networks and Heterogeneous Media 2(4) 749-757.

Bayen, A, R Raffard, C Tomlin. 2004. Network congestion alleviation using adjoint hybrid control: Application to highways. R Alur, G Pappas, eds., Hybrid Systems: Computation and Control. 95-110.

Cassidy, M, S Ahn. 2005. Driver turn-taking behavior in congested freeway merges. Transportation Research Record 1934 140-147.

Coclite, G, M Garavello, B Piccoli. 2005. Traffic flow on a road network. SIAM Journal on Mathematical Analysis 36(6) 1862-1886. URL http://link.aip.org/link/?SJM/36/1862/1.

Coclite, GM, B Piccoli. 2002. Traffic flow on a road network. ArXiv Mathematics e-prints URL http: //adsabs.harvard.edu/abs/2002math......2146C.

Daganzo, C F. 1996. The nature of freeway gridlock and how to prevent it. J. B. Lesort, ed., 13th Int. Symp. on Transportation and Traffic Theory. Elsevier, New York, 629-646.

Gugat, M, M Herty, A Klar, G Leugering. 2005. Optimal control for traffic flow networks. Journal of Optimization Theory and Applications 126(3) 589-616.

Jin, WL, HM Zhang. 2003. On the distribution schemes for determining flows through a merge. Transportation Research Part B 37(6) 521-540.

Jin, WL, HM Zhang. 2004. A multicommodity kinematic wave simulation model of network traffic flow. Transportation Research Record 1883 59-67.

Laval, J A, C F Daganzo. 2006. Lane-changing in traffic streams. Transportation Research Part B 40(3) 251-264.

Laval, J A, L Leclercq. 2008. Microscopic modeling of the relaxation phenomenon using a macroscopic lane-changing model. Transportation Research Part B 42(6) 511-522. URL http://dx.doi.org/10. 1016/j.trb.2007.10.004.

Leclercq, L, J Laval, E Chevallier. 2007. The Lagrangian coordinate system and what it means for first order traffic flow models. B Heydecker, M Bell, R Allsop, eds., 17th International Symposium on Transportation and Traffic Theory. Elsevier, New York.

LeVeque, R L. 1993. Numerical methods for conservation laws. Birkhauser Verlag .

Lighthill, M J, GB Whitham. 1955. On kinematic waves. I Flow movement in long rivers. II A theory of traffic flow on long crowded roads. Proc. Roy. Soc. 229(A) 281-345.

Richards, P I. 1956. Shockwaves on the highway. Operations Research (4) 42-51. 

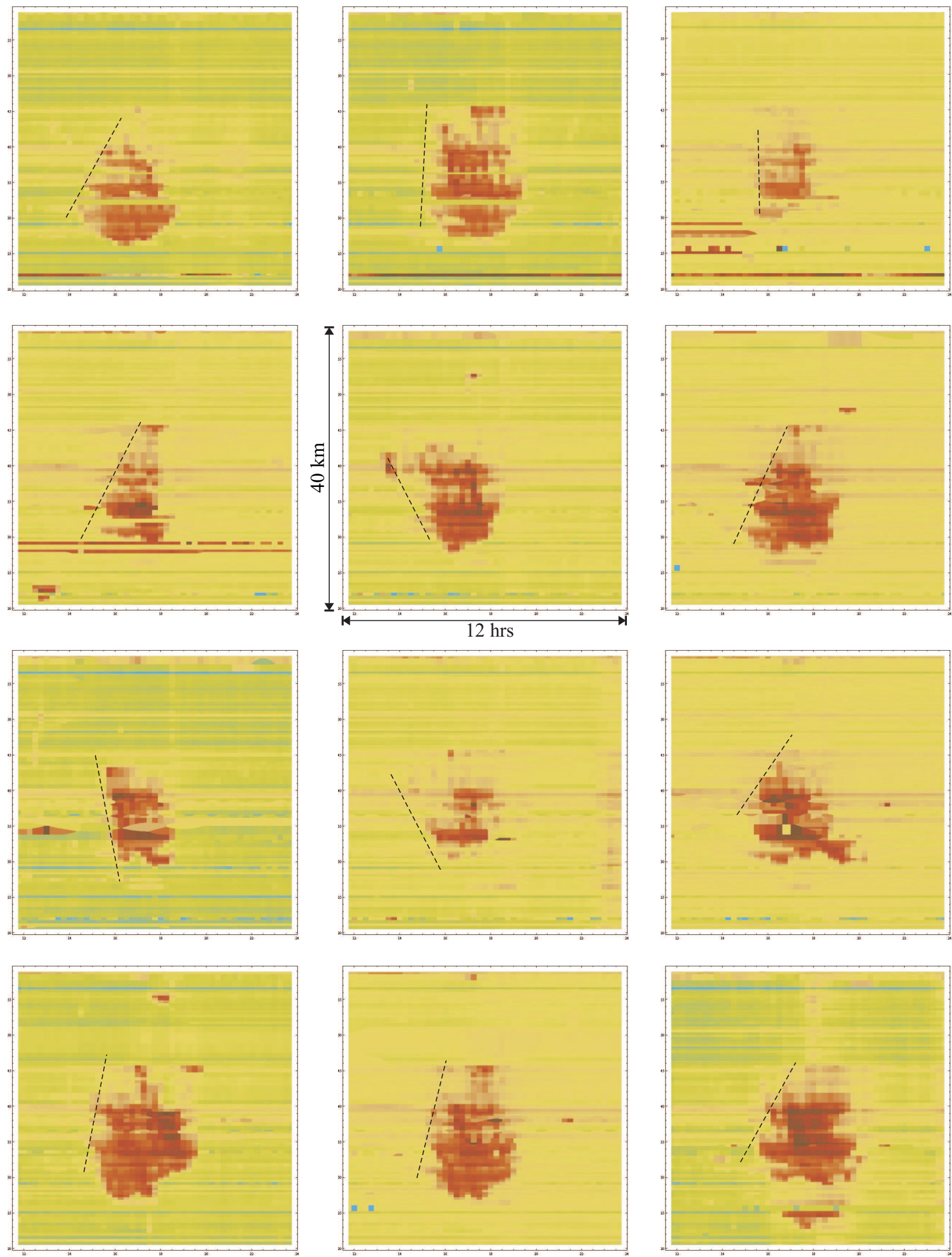

Figure 4 Density map from a $40 \mathrm{~km}$ corridor on the I-285 freeway in Atlanta, Georgia. This data corresponds to the period noon-midnight, all lanes combined, eastbound direction for selected days in January 2008. The dashed lines indicate the direction of the free-flow to congestion transitions A-C in the text. 


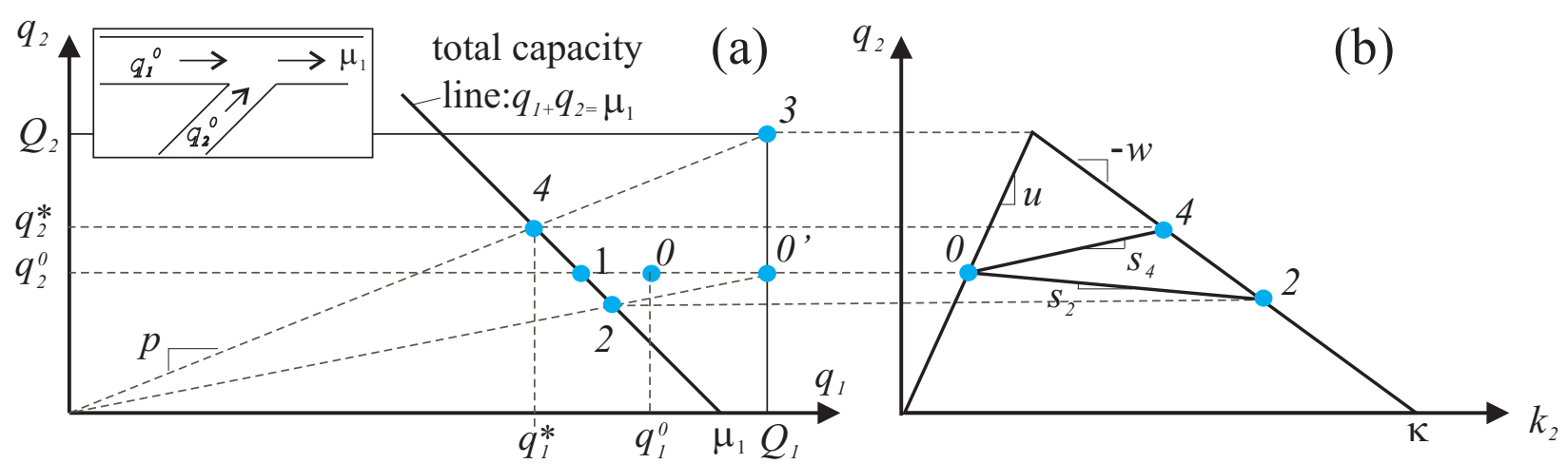

Figure 5 (a) Daganzo's merge diagram; (b) fundamental diagram on approach 2. 